PROCEEDINGS OF THE

AMERICAN MATHEMATICAL SOCIETY

Volume 129, Number 1, Pages 271-278

S 0002-9939(00)05505-2

Article electronically published on June 14, 2000

\title{
EXTENDING BAIRE PROPERTY BY COUNTABLY MANY SETS
}

\author{
PIOTR ZAKRZEWSKI
}

(Communicated by Carl G. Jockusch, Jr.)

\begin{abstract}
We prove that if ZFC is consistent so is ZFC + "for any sequence $\left(A_{n}\right)$ of subsets of a Polish space $\langle X, \tau\rangle$ there exists a separable metrizable topology $\tau^{\prime}$ on $X$ with $\mathbf{B}(X, \tau) \subseteq \mathbf{B}\left(X, \tau^{\prime}\right), \operatorname{MGR}\left(X, \tau^{\prime}\right) \cap \mathbf{B}(X, \tau)=$ $\operatorname{MGR}(X, \tau) \cap \mathbf{B}(X, \tau)$ and $A_{n}$ Borel in $\tau^{\prime}$ for all $n$." This is a category analogue of a theorem of Carlson on the possibility of extending Lebesgue measure to any countable collection of sets. A uniform argument is presented, which gives a new proof of the latter as well.

Some consequences of these extension properties are also studied.
\end{abstract}

\section{INTRODUCTION}

Most of our terminology and notation is standard and essentially agrees with [10]. Throughout the paper $(X, \tau)$ is a fixed perfect Polish space.

The results of this article are the category analogues of some known theorems on extending a given finite, countably additive, atomless measure $\mu$ defined on the $\sigma$-algebra $\mathbf{B}(X, \tau)$ (denoted also by $\mathbf{B}(X)$ ) of Borel subsets of $X$ (shortly: a Borel measure) to a countably additive measure $m^{\prime}$ defined on a $\sigma$-algebra $\mathcal{A}$ containing $\mathbf{B}(X)$ and such that $\mu^{\prime} \mid \mathcal{A}=\mu$ (it is well-known that without loss of generality $\mu$ can be assumed to be the Lebesgue measure on the reals - see [10, 17.41).

Loś and Marczewski [11] proved that if $\mathcal{A}$ is generated (as a $\sigma$-algebra) by $\mathbf{B}(X)$ plus any finite collection of new subsets of $X$, then such an extension exists. Banach and Kuratowski [1] showed that assuming $\mathrm{CH}$ there is a countable collection of subsets of $X$ which cannot be included in the domain of any extension of $\mu$. On the other hand, Solovay [14] proved that the possibility of extending $\mu$ to $\mathcal{P}(X)$, the power set of $X$, is equiconsistent with the existence of a measurable cardinal. Finally, Carlson [4] obtained the consistency of "if $\mathcal{A}$ is generated by $\mathbf{B}(X)$ plus any countable collection of new subsets of $X$, then there is an extension of $\mu$ defined on $\mathcal{A} "$.

Observe that we can formulate results about extensions of measures in terms of the related $\sigma$-ideals and quotient $\sigma$-algebras. Let $\mathrm{NULL}_{\mu}$ denote the $\sigma$-ideal of all subsets of $X$, having outer measure zero with respect to a given Borel measure $\mu$ on $X$. For an infinite cardinal $\kappa$ let $\mathrm{R}_{\kappa}$ denote the measure algebra of $2^{\kappa}$, i.e., the

Received by the editors July 10, 1998 and, in revised form, March 16, 1999.

2000 Mathematics Subject Classification. Primary 03E35, 54E52; Secondary 28A05.

Key words and phrases. Measure and category, Borel sets, Baire Property, $\sigma$-algebra, $\sigma$-ideal.

The author was partially supported by KBN grant 2 P03A 04709 and by the Alexander von Humboldt Foundation. 
$\sigma$-algebra generated by the basic open sets modulo null sets with respect to the usual product measure.

Proposition 1.1 (folklore?). Let $\mu$ be a finite, atomless Borel measure on $X$. For a $\sigma$-algebra $\mathcal{A}$ containing $\mathbf{B}(X)$ the following are equivalent:

(i) There exists an extension of $\mu$ to a measure defined on $\mathcal{A}$.

(ii) There exists a $\sigma$-ideal $\mathcal{I}$ in $\mathcal{A}$ such that:

1. $\mathcal{I} \cap \mathbf{B}(X)=\mathrm{NULL}_{\mu} \cap \mathbf{B}(X)$;

2. The Boolean algebra $\mathcal{A} / \mathcal{I}$ can be completely embedded in the algebra $R_{\kappa}$ for some infinite cardinal $\kappa$.

Moreover, if $\mathcal{A}$ is countably generated, then condition 2 can be replaced by: $2^{\prime} . \mathcal{A} / \mathcal{I} \cong R_{\omega}$.

Proof. (i) $\Rightarrow$ (ii). Just let $\mathcal{I}=\left\{A \in \mathcal{A}: \mu^{\prime}(A)=0\right\}$, where $\mu^{\prime}$ is a given extension of $\mu$ defined on $\mathcal{A}$. Then condition 1 is obvious and Maharam's theorem (see [5]) implies the rest.

(ii) $\Rightarrow$ (i). Let $\nu$ be a probability measure on $\mathcal{A}$ with $\mathcal{I}=\{A \in \mathcal{A}: \nu(A)=0\}$. Then the Radon-Nikodym theorem tells us that there is a Borel function $f$ on $X$ such that

$$
\mu(B)=\int_{B} f d \nu, \text { for every } B \in \mathbf{B}(X) .
$$

Hence the formula

$$
\mu^{\prime}(B)=\int_{B} f d \nu, \text { for } B \in \mathcal{A},
$$

defines an extension of $\mu$ to a measure $\mu^{\prime}$ on $\mathcal{A}$.

Finally, the "moreover" part follows from the fact that any atomless countably completely generated complete subalgebra of $\mathrm{R}_{\kappa}$ is isomorphic to $\mathrm{R}_{\omega}$ (see [5]).

This motivates the following definition. Let $\operatorname{MGR}(X, \tau)$ (or simply $\operatorname{MGR}(X)$ ) be the $\sigma$-ideal of all meager subsets of $X$. For an infinite cardinal $\kappa$ let $\mathrm{C}_{\kappa}$ denote the category algebra of $2^{\kappa}$, i.e., the $\sigma$-algebra generated by the basic open sets modulo meager sets.

Definition 1.2. Suppose $\mathcal{A}$ is a $\sigma$-algebra containing $\mathbf{B}(X)$. We say that the Baire Property can be extended to $\mathcal{A}$, if there exists a $\sigma$-ideal $\mathcal{I}$ in $\mathcal{A}$ such that:

1. $\mathcal{I} \cap \mathbf{B}(X)=\operatorname{MGR}(X) \cap \mathbf{B}(X)$;

2. The Boolean algebra $\mathcal{A} / \mathcal{I}$ can be completely embedded in the algebra $\mathrm{C}_{\kappa}$ for some infinite cardinal $\kappa$.

Kamburelis 9] proved that the possibility of extending the Baire Property to $\mathcal{P}(X)$ is equiconsistent with the existence of a measurable cardinal. When $\mathcal{A}$ is countably generated, which is the case dealt with in this note, the definition above takes a nicer form. Recall that $\mathrm{C}_{\omega}$ is the unique, up to an isomorphism, complete, atomless Boolean algebra with a countable dense subset.

Proposition 1.3. If $\mathcal{A}$ is a countably generated $\sigma$-algebra containing $\mathbf{B}(X, \tau)$, then the following are equivalent:

(i) The Baire Property can be extended to $\mathcal{A}$;

(ii) There exists a $\sigma$-ideal $\mathcal{I}$ in $\mathcal{A}$ such that:

1. $\mathcal{I} \cap \mathbf{B}(X, \tau)=\operatorname{MGR}(X, \tau) \cap \mathbf{B}(X, \tau)$,

2. $\mathcal{A} / \mathcal{I} \cong C_{\omega}$; 
(iii) There exists a separable metrizable topology $\tau^{\prime}$ on $X$ with $\mathcal{A}=\mathbf{B}\left(X, \tau^{\prime}\right)$ and $\operatorname{MGR}\left(X, \tau^{\prime}\right) \cap \mathbf{B}(X, \tau)=\operatorname{MGR}(X, \tau) \cap \mathbf{B}(X, \tau)$.

Proof. (i) $\Rightarrow$ (ii) follows from the fact that any atomless countably completely generated complete subalgebra of $\mathrm{C}_{\kappa}$ is isomorphic to $\mathrm{C}_{\omega}$.

To get (ii) $\Rightarrow$ (iii) it suffices to prove that if $\mathcal{I}$ is a $\sigma$-ideal in a countably generated $\sigma$-algebra $\mathcal{A}$ that separates points in $X$ and $\mathcal{A} / \mathcal{I} \cong \mathrm{C}_{\omega}$, then there exists a separable metrizable topology $\tau^{\prime}$ on $X$ with $\mathcal{A}=\mathbf{B}\left(X, \tau^{\prime}\right)$ and $\mathcal{I}=\operatorname{MGR}\left(X, \tau^{\prime}\right) \cap \mathcal{A}$.

First note that since $\mathcal{A}$ is countably generated and separates points in $X$, there is a separable metric $d$ on $X$ such that $\mathcal{A}=\mathbf{B}(X, d)$ (see [10, 12.1). Let $(\hat{X}, \hat{d})$ be the completion of $(X, d)$. Define a $\sigma$-ideal $J$ in $\mathbf{B}(\hat{X}, \hat{d})$ by:

$$
A \in J \Longleftrightarrow A \cap X \in \mathcal{I} \text {, for } A \in \mathbf{B}(\hat{X}, \hat{d}) .
$$

Now, since

$$
\mathbf{B}(\hat{X}, \hat{d}) / J \cong \mathcal{A} / \mathcal{I} \cong \mathrm{C}_{\omega},
$$

there is a Polish topology $\tau_{1}$ on $\hat{X}$ for which we have $\mathbf{B}(\hat{X}, \hat{d})=\mathbf{B}\left(\hat{X}, \tau_{1}\right)$ and $J=\operatorname{MGR}\left(\hat{X}, \tau_{1}\right) \cap \mathbf{B}\left(\hat{X}, \tau_{1}\right)$ (see [10], 15.10). Let $\tau^{\prime}=\tau_{1} \mid X$ be the relative topology on $X$ treated as a subspace of $\left(\hat{X}, \tau_{1}\right)$. Since, clearly, $\mathcal{A}=\mathbf{B}\left(X, \tau^{\prime}\right)$, to complete the proof it is enough to check that $\mathcal{I}=\operatorname{MGR}\left(X, \tau^{\prime}\right) \cap \mathcal{A}$. In view of the definitions of the $\sigma$-ideals involved, it suffices to prove that for any $A \in \mathbf{B}\left(\hat{X}, \tau_{1}\right)$

$$
A \in \operatorname{MGR}\left(\hat{X}, \tau_{1}\right) \Longleftrightarrow A \cap X \in \operatorname{MGR}\left(X, \tau^{\prime}\right) .
$$

This, in turn, reduces to the fact that if $A$ is closed in $\tau_{1}$, then it is nowhere dense in $\tau_{1}$ if and only if $A \cap X$ is nowhere dense in $\tau^{\prime}$. But for the latter note that if $U \in \tau_{1}$ and $U \cap X=\emptyset$, then $U \in J=\operatorname{MGR}\left(\hat{X}, \tau_{1}\right)$, so $U=\emptyset$.

Finally, (ii) $\Rightarrow$ (i) is trivial and (iii) $\Rightarrow$ (ii) follows from the fact that, if $\tau^{\prime}$ is a second countable topology on $X$ with no isolated points and $X \notin \operatorname{MGR}\left(X, \tau^{\prime}\right)$, then

$$
\mathbf{B}\left(X, \tau^{\prime}\right) /\left(\operatorname{MGR}\left(X, \tau^{\prime}\right) \cap \mathbf{B}\left(X, \tau^{\prime}\right)\right) \cong \mathrm{C}_{\omega}
$$

The main result of this paper shows that it is consistent, relative to the consistency of ZFC, that the Baire Property can be extended to any countably generated $\sigma$-algebra $\mathcal{A}$ containing $\mathbf{B}(X)$. In Section 2 we show that the latter follows from a statement concerning special sets of reals, a dual version of which implies that any Borel measure on $X$ can be extended to a measure on such an $\mathcal{A}$. In Section 3 we indicate some consequences of the extension properties in question.

In the sequel $I$ always stands either for $\operatorname{MGR}(X)$ or $\mathrm{NULL}_{\mu}$ for a given Borel measure $\mu$ on $X$.

\section{Main Results}

Recall that an $I$-Lusin set is an uncountable subset of $X$ which has countable intersection with every element of $I$. If $I=\operatorname{MGR}(X)\left(I=\mathrm{NULL}_{\mu}\right.$, resp. $)$, then an $I$-Lusin set is called a Lusin set (a Sierpiński set, resp.).

We shall need two auxiliary lemmas. The first one was proved by Recław and the author in [13] (see [13], Lemma 3.2). 
Lemma 2.1. Let $\mathcal{A}$ be any countably generated $\sigma$-algebra containing $\mathbf{B}(X)$.

If there exists a I-Lusin set of cardinality the continuum and every subset of $X$ of cardinality the continuum contains a one-to-one Borel image of a set not in $I$, then there exist a set $Z \subseteq X, Z \notin I$, and a $\mathbf{B}(Z)-\mathcal{A}$ measurable function $\varphi: Z \rightarrow X$ such that:

1. $\forall A \in I \varphi^{-1}[A] \in I$,

2. $\forall A \in(\mathbf{B}(X) \backslash I) \varphi^{-1}[A] \notin I$.

Lemma 2.2. Suppose that $Z \subseteq X$ and $Z \notin I$. Then

$$
\mathbf{B}(Z) /(I \cap \mathbf{B}(Z)) \cong \mathbf{B}(X) /(I \cap \mathbf{B}(X)) .
$$

Proof. Let $Z^{*} \in \mathbf{B}(X)$ be such that $Z \subseteq Z^{*}$ and $\forall B \in \mathbf{B}(X) B \subseteq Z^{*} \backslash Z \Rightarrow B \in I$.

Note that the function $F: \mathbf{B}(Z) /(I \cap \mathbf{B}(Z)) \rightarrow \mathbf{B}\left(Z^{*}\right) /\left(I \cap \mathbf{B}\left(Z^{*}\right)\right)$ given by

$$
F\left([B \cap Z]_{I \cap \mathrm{B}(Z)}\right)=\left[B \cap Z^{*}\right]_{I \cap \mathrm{B}\left(Z^{*}\right)} \text { for } B \in \mathbf{B}(X)
$$

is an isomorphism of the corresponding Boolean algebras.

But the algebras $\mathbf{B}\left(Z^{*}\right) /\left(I \cap \mathbf{B}\left(Z^{*}\right)\right)$ and $\mathbf{B}(X) /(I \cap \mathbf{B}(X))$ are isomorphic, since the latter is homogeneous.

Now we are ready to state the main result.

Theorem 2.3. Let $\mathcal{A}$ be any countably generated $\sigma$-algebra containing $\mathbf{B}(X)$.

If there exists a I-Lusin set of cardinality the continuum and every subset of $X$ of cardinality the continuum contains a one-to-one Borel image of a set not in $I$, then there exists a $\sigma$-ideal $\mathcal{I}$ in $\mathcal{A}$ such that:

1. $\mathcal{I} \cap \mathbf{B}(X)=I \cap \mathbf{B}(X)$,

2. $\mathcal{A} / \mathcal{I} \cong \mathbf{B}(X) /(I \cap \mathbf{B}(X))$.

In particular, if there exists a Lusin set (a Sierpinski set, resp.) of cardinality the continuum and every set of reals of cardinality the continuum contains a oneto-one Borel image of a non-meager set (a set of positive outer Lebesgue measure, resp.), then the Baire Property (the Lebesgue measure, resp.) can be extended to any countably generated $\sigma$-algebra containing Borel sets.

Proof. Let $Z \notin I$ and $\varphi: Z \rightarrow X$ be the objects whose existence is guaranteed by Lemma 2.1. Define $\mathcal{I}$ by letting

$$
A \in \mathcal{I} \Longleftrightarrow \phi^{-1}[A] \in I, \text { for } A \in \mathcal{A} \text {. }
$$

Condition (1) is clearly satisfied. Moreover, $\phi$ induces a complete embedding of the Boolean algebra $\mathcal{A} / \mathcal{I}$ into the algebra $\mathbf{B}(Z) /(I \cap \mathbf{B}(Z))$ which in turn, by Lemma 2.2, is isomorphic to $\mathbf{B}(X) /(I \cap \mathbf{B}(X))$. It follows that the algebra $\mathcal{A} / \mathcal{I}$ is also isomorphic to $\mathbf{B}(X) /(I \cap \mathbf{B}(X))$ as an atomless, complete subalgebra of the latter.

If $I=\operatorname{MGR}(X)\left(I=\mathrm{NULL}_{\mu}\right.$, resp.), then the hypotheses of Theorem 2.3 are true in the Cohen real model (random real model, resp.), i.e., the model obtained by adding $\kappa>\omega_{1}$ Cohen reals (random reals, resp.) to a model of $\mathrm{CH}$ (see Miller [12]). The natural question, to what extent these conditions are also necessary, will be briefly discussed in the next section. 


\section{Corollaries AND REMARKS}

It seems to be of some interest to study consequences of statements like "Lebesgue measure can be extended by any countable collection of sets" and its category counterpart, dealt primarily with in this paper. The first one implies for instance that if $Z \subseteq X, Z \notin$ NULL $_{\mu}$ and a family $\left\{D^{y}: y \in Z\right\}$ consists of non-null measurable sets, then there exists $E \subseteq Z, E \notin \mathrm{NULL}_{\mu}$ such that $\bigcap_{y \in E} D^{y} \neq \emptyset$ (see [15]). Dually, we have (compare [8], Proposition 2, where a different proof of a similar result is given).

Theorem 3.1. Assume that the Baire Property can be extended to any countably generated $\sigma$-algebra containing $\mathbf{B}(X)$.

If $Z \subseteq X, Z \notin \operatorname{MGR}(X)$ and a family $\left\{D^{y}: y \in Z\right\}$ consists of non-meager sets with the Baire Property, then there exists $E \subseteq Z, E \notin \operatorname{MGR}(X)$ such that $\bigcap_{y \in E} D^{y} \neq \emptyset$.

Proof. Equivalently, we shall prove that given a set $D \subseteq X \times X$ with all sections $D_{x}=\{y:\langle x, y\rangle \in D\}$ meager and all sections $D^{y}=\{x:\langle x, y\rangle \in D\}$ having the Baire Property, then $D^{y} \in \operatorname{MGR}(X)$ for every $y$ outside a meager set.

To begin with, cover $D$ by a set $C$ with $C_{x} \in \mathrm{F}_{\sigma} \cap \operatorname{MGR}(X)$ for every $x \in X$. It is easy to see that $C \in \mathcal{A} \otimes \mathbf{B}(X)$ for a certain countably generated $\sigma$-algebra $\mathcal{A}$. Since the Baire Property can be extended to $\mathcal{A}$, we have $\mathcal{A}=\mathbf{B}\left(X, \tau^{\prime}\right)$ for a certain second countable topology $\tau^{\prime}$ on $X$ with $\operatorname{MGR}\left(X, \tau^{\prime}\right) \cap \mathbf{B}(X, \tau)=\operatorname{MGR}(X, \tau) \cap \mathbf{B}(X, \tau)$.

Now the Kuratowski-Ulam theorem (see [10, 8.41), applied to $C$ considered as a Borel subset of the product of the spaces $\left(X, \tau^{\prime}\right)$ and $(X, \tau)$, gives

$$
\left\{y \in X: C^{y} \notin \operatorname{MGR}\left(X, \tau^{\prime}\right)\right\} \in \operatorname{MGR}(X, \tau) .
$$

But

$$
\left\{y: D^{y} \notin \operatorname{MGR}(X, \tau)\right\} \subseteq\left\{y \in X: C^{y} \notin \operatorname{MGR}\left(X, \tau^{\prime}\right)\right\},
$$

due to the fact that $\operatorname{MGR}\left(X, \tau^{\prime}\right) \cap \mathbf{B}(X, \tau)=\operatorname{MGR}(X, \tau) \cap \mathbf{B}(X, \tau)$ and $D^{y}$ has the Baire Property in $(X, \tau)$, for every $y \in X$.

Theorem 3.1 and its dual may be considerably generalized, if we take into account the results obtained by Recław and the author in [13]. The point is that the technical condition expressed in Lemma 2.1 turns out to be an equivalent formulation of the extension property in question. More precisely, we have

Proposition 3.2. Let $\mathcal{A}$ be a countably generated $\sigma$-algebra containing $\mathbf{B}(X)$. The following are equivalent:

(i) There exists a $\sigma$-ideal $\mathcal{I}$ in $\mathcal{A}$ such that:

1. $\mathcal{I} \cap \mathbf{B}(X)=I \cap \mathbf{B}(X)$,

2. $\mathcal{A} / \mathcal{I} \cong \mathbf{B}(X) /(I \cap \mathbf{B}(X))$.

(ii) There exist a set $Z \subseteq X, Z \notin I$, and a $\mathbf{B}(Z)-\mathcal{A}$ measurable function $\varphi$ : $Z \rightarrow X$ such that:

1. $\forall A \in I \varphi^{-1}[A] \in I$,

2. $\forall A \in(\mathbf{B}(X) \backslash I) \varphi^{-1}[A] \notin I$.

Proof. Only the implication (i) $\Rightarrow$ (ii) requires proof, since the other one was established in the course of proving Theorem 2.3. 
As in the proof of Proposition $1.3(($ ii $) \Rightarrow$ (iii)) find a separable metric $d$ on $X$ such that $\mathcal{A}=\mathbf{B}(X, d)$, let $(\hat{X}, \hat{d})$ be the completion of $(X, d)$ and define a $\sigma$-ideal $J$ in $\mathbf{B}(\hat{X}, \hat{d})$ by:

$$
A \in J \Longleftrightarrow A \cap X \in \mathcal{I}, \text { for } A \in \mathbf{B}(\hat{X}, \hat{d})
$$

Since

$$
\mathbf{B}(\hat{X}, \hat{d}) / J \cong \mathcal{A} / \mathcal{I} \cong \mathbf{B}(X, \tau) /(I \cap \mathbf{B}(X, \tau))
$$

there is a Borel isomorphism $\psi: \hat{X} \rightarrow X$ between $(\hat{X}, \hat{d})$ and $(X, \tau)$ such that

$$
A \in J \Longleftrightarrow \psi[A] \in \operatorname{MGR}(X, \tau), \text { for } A \in \mathbf{B}(\hat{X}, \hat{d})
$$

(see [10], 15.10). Finally define $Z=\psi[X]$ and $\varphi=\psi^{-1} \mid Z$. It is easy to check that this works.

As corollaries we obtain the following reformulations of Theorems 3.3 and 3.4 from [13].

Theorem 3.3. Assume that the Baire Property can be extended to any countably generated $\sigma$-algebra containing $\mathbf{B}(X)$.

Let $Y$ be a Polish space and suppose that $J$ is a $\sigma$-ideal on $Y$ generated by a hereditary $\mathbf{\Pi}_{\mathbf{1}}^{\mathbf{1}}$ (in the Effros Borel structure, see [10], §35) family of closed subsets of $Y$.

If $Z \subseteq Y, Z \notin J$, and a family $\left\{D^{y}: y \in Z\right\}$ consists of non-meager sets with the Baire Property, then there exists $E \subseteq Z, E \notin J$, such that $\bigcap_{y \in E} D^{y} \neq \emptyset$.

Theorem 3.4. Assume that the Lebesgue measure can be extended to any countably generated $\sigma$-algebra containing $\mathbf{B}(X)$.

Let $Y$ be a Polish space and suppose that $J$ is a $\sigma$-ideal on $Y$ generated by any of the following families of closed subsets of $Y$ :

(i) all compact sets (in this case $Y$ is assumed to be non- $\sigma$-compact),

(ii) all closed sets in $\mathrm{NULL}_{\nu}$ for a $\sigma$-finite Borel continuous measure $\nu$ on $Y$,

(iii) all closed subsets of a $\Pi_{1}^{1}$ set $A \subseteq Y$.

If $Z \subseteq Y, Z \notin J$, and a family $\left\{D^{y}: y \in Z\right\}$ consists of non-null measurable sets, then there exists $E \subseteq Z, E \notin J$, such that $\bigcap_{y \in E} D^{y} \neq \emptyset$.

Let us now discuss the hypotheses of Theorem 2.3. It is well-known that a set $A \subseteq X$ contains a Borel one-to-one image of a set of positive outer $\mu$-measure if and only if it is not a universal measure zero set, i.e., $A \notin \mathrm{NULL}_{\nu}$ for a certain finite atomless Borel measure $\nu$. The non-existence of universal measure zero sets of size continuum means, in turn, that if $\mathcal{A}$ is a $\sigma$-algebra generated by $\mathbf{B}(X)$ plus any countable collection of new subsets of $X$, then $\mathcal{A}$ carries a finite, countably additive measure, vanishing on singletons - see [3] (every such measure clearly extends a certain Borel measure but this does not necessarily mean that there is one which extends the measure $\mu$, fixed in advance). On the category side, sets that do not contain Borel one-to-one images of non-meager sets were studied by Grzegorek in [6], 7] and called there $\overline{\mathrm{AFC}}$ sets. One can prove that the non-existence of $\overline{\mathrm{AFC}}$ sets of size continuum means that if $\mathcal{A}$ is a countably generated $\sigma$-algebra containing $\mathbf{B}(X, \tau)$, then there exists a separable metrizable topology $\tau^{\prime}$ on $X$ such that $X$ 
is dense in itself, $\mathcal{A}=\mathbf{B}\left(X, \tau^{\prime}\right)$ and $X \notin \operatorname{MGR}\left(X, \tau^{\prime}\right)-$ see [16]. (It is easy to see that for each such topology $\tau^{\prime}$ there is a certain Polish topology $\tau^{\prime \prime}$ on $X$ such that $\mathbf{B}\left(X, \tau^{\prime \prime}\right)=\mathbf{B}(X, \tau)$ and $\operatorname{MGR}\left(X, \tau^{\prime}\right) \cap \mathbf{B}(X, \tau)=\operatorname{MGR}\left(X, \tau^{\prime \prime}\right) \cap \mathbf{B}(X, \tau)$, but this does not necessarily mean that there is one for which $\operatorname{MGR}\left(X, \tau^{\prime}\right) \cap \mathbf{B}(X, \tau)=$ $\operatorname{MGR}(X, \tau) \cap \mathbf{B}(X, \tau)$, where $\tau$ is the Polish topology fixed in advance.)

In view of the above we see that the non-existence of $\overline{\mathrm{AFC}}$ sets (universal measure zero sets, resp.) of size continuum is a necessary condition for the respective extension principles and it seems conceivable that it is also sufficient. The latter, however, is not the case.

Proposition 3.5. The following two statements are consistent with $Z F C$ :

(i) There are no $\overline{A F C}$ sets of size continuum but for any Polish topology $\tau$ on $X$ there is a countably generated $\sigma$-algebra $\mathcal{A}$ containing $\mathbf{B}(X, \tau)$ such that the Baire Property cannot be extended to $\mathcal{A}$.

(ii) There are no universal measure zero sets of size continuum but for any finite, atomless Borel measure $\mu$ on $X$ there is a countably generated $\sigma$-algebra $\mathcal{A}$ containing $\mathbf{B}(X)$ such that there is no extension of $\mu$ to a measure defined on $\mathcal{A}$.

Proof. A model for both, (i) and (ii), is the iterated perfect set model, i.e., the model obtained by the countable support iteration of length $\omega_{2}$ of the Sacks forcing over a model of $\mathrm{CH}$. We give an argument for the category case only - the measure case is symmetric.

So let us work in this model. Then, by a result of Miller [12], there are no $\overline{\mathrm{AFC}}$ sets of size continuum. On the other hand, $X$ is the union of $\omega_{1}$ many meager sets (see [2], 7.6.2). Using this, it is easy to construct a family $\left\{D^{y}: y \in X\right\}$ of co-meager sets such that for every $E \notin \operatorname{MGR}(X), \bigcap_{y \in E} D^{y} \in \operatorname{MGR}(X)$. Now the second clause of (i) follows from Theorem 3.1.

In order to complete the discussion of the duality between measure and category, as far as the extension properties dealt with in this paper are concerned, let us remark that category versions of both the Łoś-Marczewski and the BanachKuratowski theorems are also true.

For the latter, just take the entries of an Ulam $\left(\omega_{1}, \omega\right)$-matrix, which under $\mathrm{CH}$ can be identified with sets of reals. It is well-known that together with Borel sets they generate a countably generated $\sigma$-algebra, say $\mathcal{A}$, with the property that for no $\sigma$-ideal $\mathcal{I}$ in $\mathcal{A}$ containing singletons does the Boolean algebra $\mathcal{A} / \mathcal{I}$ satisfy the countable chain condition. Hence neither Lebesgue measure nor Baire Property can be extended to $\mathcal{A}$.

Finally, the fact that if $\mathcal{A}$ is generated by $\mathbf{B}(X)$ plus any finite collection of subsets of $X$, then the Baire Property can be extended to $\mathcal{A}$ may be established by a simple argument that covers the measure case as well.

Proposition 3.6. If a $\sigma$-algebra $\mathcal{A}$ is generated by $\mathbf{B}(X)$ plus a finite collection $\left\{Z_{1}, \ldots, Z_{n}\right\}$ of subsets of $X$, then there exists a $\sigma$-ideal $\mathcal{I}$ in $\mathcal{A}$ such that:

1. $\mathcal{I} \cap \mathbf{B}(X)=I \cap \mathbf{B}(X)$,

2. $\mathcal{A} / \mathcal{I} \cong \mathbf{B}(X) /(I \cap \mathbf{B}(X))$.

Proof. Without loss of generality we can assume that $\left\{Z_{1}, \ldots, Z_{n}\right\}$ is a partition of $X$ into sets not in $I$. Now it suffices to let $\mathcal{I}=I \cap \mathcal{A}$. Indeed, we have

$$
\mathcal{A} / \mathcal{I} \cong \mathbf{B}\left(Z_{1}\right) /\left(I \cap \mathbf{B}\left(Z_{1}\right)\right) \times \cdots \times \mathbf{B}\left(Z_{n}\right) /\left(I \cap \mathbf{B}\left(Z_{n}\right)\right) .
$$


But

$$
\begin{aligned}
\mathbf{B}\left(Z_{1}\right) /(I & \left.\cap \mathbf{B}\left(Z_{1}\right)\right) \times \cdots \times \mathbf{B}\left(Z_{n}\right) /\left(I \cap \mathbf{B}\left(Z_{n}\right)\right) \\
& \cong \mathbf{B}(X) /(I \cap \mathbf{B}(X)) \times \cdots \times \mathbf{B}(X) /(I \cap \mathbf{B}(X)) \cong \mathbf{B}(X) /(I \cap \mathbf{B}(X)),
\end{aligned}
$$

due to Lemma 2.2 and the homogeneity of the algebra $\mathbf{B}(X) /(I \cap \mathbf{B}(X))$.

\section{REFERENCES}

1. S. Banach and K. Kuratowski, Sur une généralization du problème de la mesure, Fund. Math. 14 (1929), 127-131.

2. T. Bartoszyński, H. Judah, Set theory: on the structure of the real line, A K Peters, Wellesley 1995. MR 96k:03002

3. J. Brown, G. Cox, Classical theory of totally imperfect spaces, Real Analysis Exchange 7 (1981-2), 185-232. MR 84g:54021

4. T. Carlson, Extending Lebesgue measure by infinitely many sets, Pac. J. Math. 115 (1984), 33-45. MR 86a:03055

5. D. H. Fremlin, Measure algebras, in Handbook of Boolean algebras, North-Holland, 1989, 876-980. CMP 21:10

6. E. Grzegorek Always of the first category sets, Rend. Circ. Mat. Palermo, II. Ser. Suppl. 6 (1984), 139-147. CMP 17:10

7. E. Grzegorek Always of the first category sets. II, Rend. Circ. Mat. Palermo, II. Ser. Suppl. 10 (1985), 43-48. MR 88j:54054

8. A. Kamburelis, A new proof of the Gitik-Shelah theorem, Israel J. Math. 72 (1990), 373-380. MR 92h:03069

9. A. Kamburelis, On cardinal numbers related to Baire property, preprint, Wrocław 1989.

10. A. S. Kechris, Classical descriptive set theory, Graduate Texts in Math. 156, Springer-Verlag 1995. MR 96e:03057]

11. J. Łoś and E. Marczewski, Extensions of measure, Fund. Math. 36 (1949), 267-276. MR 11:717h

12. A.W. Miller, Mapping a set of reals onto the reals, J. Symbolic Logic 48 (1983), 575-584. MR 84k:03125

13. I. Recław and P. Zakrzewski, Strong Fubini properties of ideals, Fund. Math. 159 (1999), 135-152. CMP 99:08

14. R. M. Solovay, Real-valued measurable cardinals, in Axiomatic Set Theory, Proc. Symp. Pure Math. 13, I, AMS, Providence, Rhode Island 1971, 397-428. MR 45:55

15. P. Zakrzewski, Strong Fubini axioms from measure extension axioms, Comment. Math. Univ. Carolinae 33,2 (1992), 291-297. MR 93k:03047

16. P. Zakrzewski, Universally meager sets, Proc. Amer. Math. Soc., to appear.

Institute of Mathematics, University of Warsaw, ul. Banacha 2, 02-097 Warsaw, POLAND

E-mail address: piotrzak@mimuw.edu.pl 